\title{
An Intelligent Manufacturing Cell Based on Human- robot Collaboration of Frequent Task Learning for Flexible Manufacturing
}

\section{Shuai Zhang ( $\square$ zhangshuaihenan@163.com )}

Zhejiang University

\section{Shiqi Li}

Huazhong University of Science and Technology

\section{Haipeng Wang}

Huazhong University of Science and Technology

\section{Xiao Li}

Huazhong University of Science and Technology

\section{Research Article}

Keywords: intelligent manufacturing cell, human-robot collaboration, task adjustment, flexible manufacturing

Posted Date: July 22nd, 2021

DOI: https://doi.org/10.21203/rs.3.rs-678197/v1

License: (c) (i) This work is licensed under a Creative Commons Attribution 4.0 International License. Read Full License

Version of Record: A version of this preprint was published at The International Journal of Advanced Manufacturing Technology on April 2nd, 2022. See the published version at https://doi.org/10.1007/s00170-022-09005-6. 


\title{
An Intelligent Manufacturing Cell based on Human-robot Collaboration of Frequent Task Learning for Flexible Manufacturing
}

\author{
Shuai Zhang ${ }^{\mathrm{a}}$, Shiqi $\mathrm{Li}^{\mathrm{b}, *}$, Haipeng Wang ${ }^{\mathrm{b}}$, Xiao $\mathrm{Li}^{\mathrm{b}, \mathrm{c}}$ \\ ${ }^{a}$ Zhejiang University, Center for Psychological Sciences, Hangzhou 310058, China \\ ${ }^{b}$ School of Mechanical Science and Engineering, Huazhong University of Science and Technology, Wuhan, 430074, China \\ ${ }^{c}$ HUST\&UBTECH Intelligent Service Robots Joint Lab, Huazhong University of Science and Technology, Wuhan 430074, China
}

\begin{abstract}
The manufacturing industry was moving towards the trend of short run production and personalized customization. That results in the challenge of the efficiency of task adjustment and the complexity of tasks for robots. Thus, this paper developed the intelligent manufacturing cell based on human-robot collaboration(HRC-IMC), combining the intelligence of cobot with that of human. And the intelligent manufacturing cell was composed with the modules of imitating learning, human-robot safety planning, task planning and visual inferring. Moreover, all modules were designed to provide a set of systematic and effective method which can improve the efficiency of task planning and new task learning. The experimental results indicated that the the efficiency of task adjustment of HRC-IMC can be increased $42.8 \%$ than that of Moveit. All in all, this study is of great significance for improving the efficiency of new task planning of cobots by digitizing the manipulation experience of human.
\end{abstract}

Keywords: intelligent manufacturing cell, human-robot collaboration, task adjustment, flexible manufacturing

\section{Introduction}

The artificial operations in production lines of manufacturing industry (like the shoemaking industry in Fig.1) were widely replaced by robots or automatic lines in recent years. To adapt to the trend of short run production and personalized customization for the flexible manufacturing like shoemaking industry, the dexterous operations of robots need to be improved and the efficiency of new task learning needs to be enhanced [1-3]. However, the current mathematical modeling for the robots' planning in production lines is very challenging when deal with new tasks, especially for dual-arm robots. And the robustness of the available mathematical models in dynamic task is low; so does the efficiency of adjustment of short run production $[4,5]$. Hence, combining the intelligence of cobot with that of human by introducing human into the manipulating cell[6, 7], which digitizes the manipulation experience of human via human-cyber physical systems and other methods, has attracted the attention of scholars again [8-10].

Human-cyber-physical systems(HCPS) is an importan$\mathrm{t}$ branch of intelligent manufacturing cell with human in the loop[11]. HCPS was developed from the concept of human in the loop cyber-physical systems[12]. Sowe, et al[13] developed cyber-physical-human systems (CPHSs) by putting human in the loop. Although they described the human capabilities using human service capability description model, few instances were conducted to verify the performance of the CPHSs. Krugh [14] tried to use the cyber-human systems(CHS) in automotive

\footnotetext{
${ }^{*}$ Corresponding author

Email address: sqli@mail .hust. edu. cn (Shiqi Li )
}

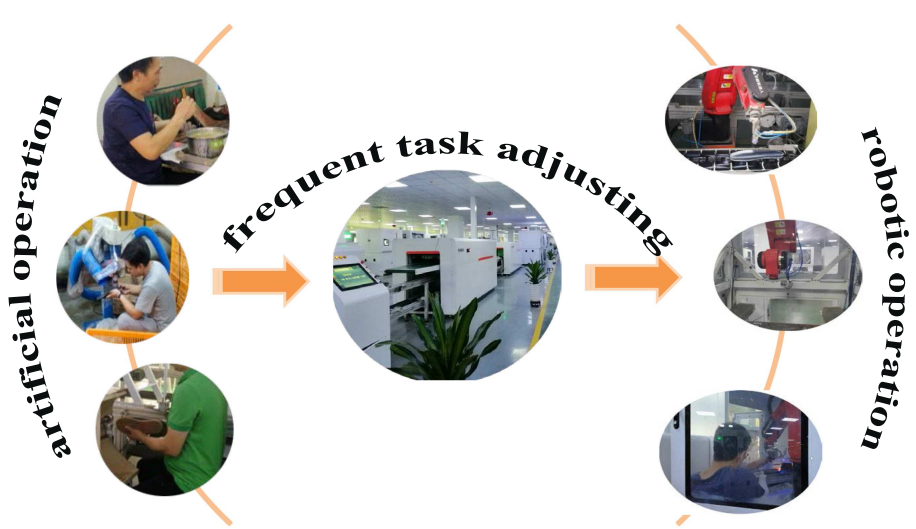

Fig. 1. The frequent task learning problems for flexible manufacturing of production line

assembly. But there are a lot of work to make the CHS in the manufacturing. Zhou, et al[15] firstly proposed the concept of HCPS. And they designed HCPS of different levels for various cases to apply HCPS in the intelligent manufacturing. Almost all of these HCPS mode systems put more emphasis on the system architecture rather than the realization of the function modules. It results in that the application of the HCPS system in specific cases seems to be impossible.

Intelligent manufacturing cell with human in the loop should contains the function module of human-robot safety, tasking planning, tasking understanding and so on. Firstly, since human and robot share the same task space in the intelligent manufacturing cell, the safety of human-robot should be ensured, which is the precondition of human working in 
the loop[16]. And the electronic fence is one of common methods for huam-robot safety in the space of human-robot coexistence[17, 18]. Besides, the distance between human and robot is treated as a critical parameter of safety planning for an electronic fence[19]. However, the distance has no significant change in close range collaborative tasks, which may result in setting numerous safety levels. Furthermore, human being overshadowed in visual sensors thereby becomes constant trouble in these tasks of human-robot coexisting in close range. Hence the prediction of humans' behavior has been attached much attention so that humans' safety can be taken into consideration in advance in the planning of robots. Since the CNNbased human pose prediction can overcome the noise shortcomings of depth sensors [20], using marker-less visual methods was widely employed [21]. In addition, some CPS based safety planning methods for enabling and controlling safe humanrobot collaborative[22, 23] are still within range of distancelevel safety strategy. And these works didn't involve the state estimation of multi-sensor fusion. So the accuracy is limited by one single sensor. Zhu L, et.al [24] integrated the spatiotemporal information of moving obstacles into the robotic motion planning to avoid collision with the human. In these methods, both humans and robots were filtered through visual algorith$\mathrm{m}$. The experimental scenario was, however, so simple that the occlusion condition was not involved. Emanuele Magrin, et.
Al [25] introduced a human-robot distance estimation cell that fused depth sensor and laser scanners in case of missing human visual data. But this distance-level safety strategy is mainly suited to the contactless interaction conditions.

The purpose of the HRC-IMC is to improve the efficiency of new task learning so as to meet the demands of short run production. So the human-guided tasking planning become another import research branch for the HRC-IMC. In the research of Edmonds, et al [26], data gloves were used to record the key points sequences of hands in the manipulation process of a bottle. In addition, objects attached with piezoelectric force transducers were employed by Pham, et al [27] to tracking grasping points (GP) in the operation process of humans. The aforementioned two methods will affect the tactile feeling of the object operated by the human hand, which may result in motion distortion [28]. Also, some grasping data sets of humans had been built for imitating the grasping of robots, such as GRAB [29], ContactPose [30] and so on. These data sets, however, were mainly composed of household items rather than objects of industrial productions. The operating habits of humans for these two kinds of objects may be in great difference.

Also, the HRC-IMC should understand the task process via visual or other sensor data[31-34]. Li, et al [35]combined the task semantic and task planning by introducing the task semantic object matrix. In this way, robots can understand the task

\section{The prototype system framework of HRC-IMC}

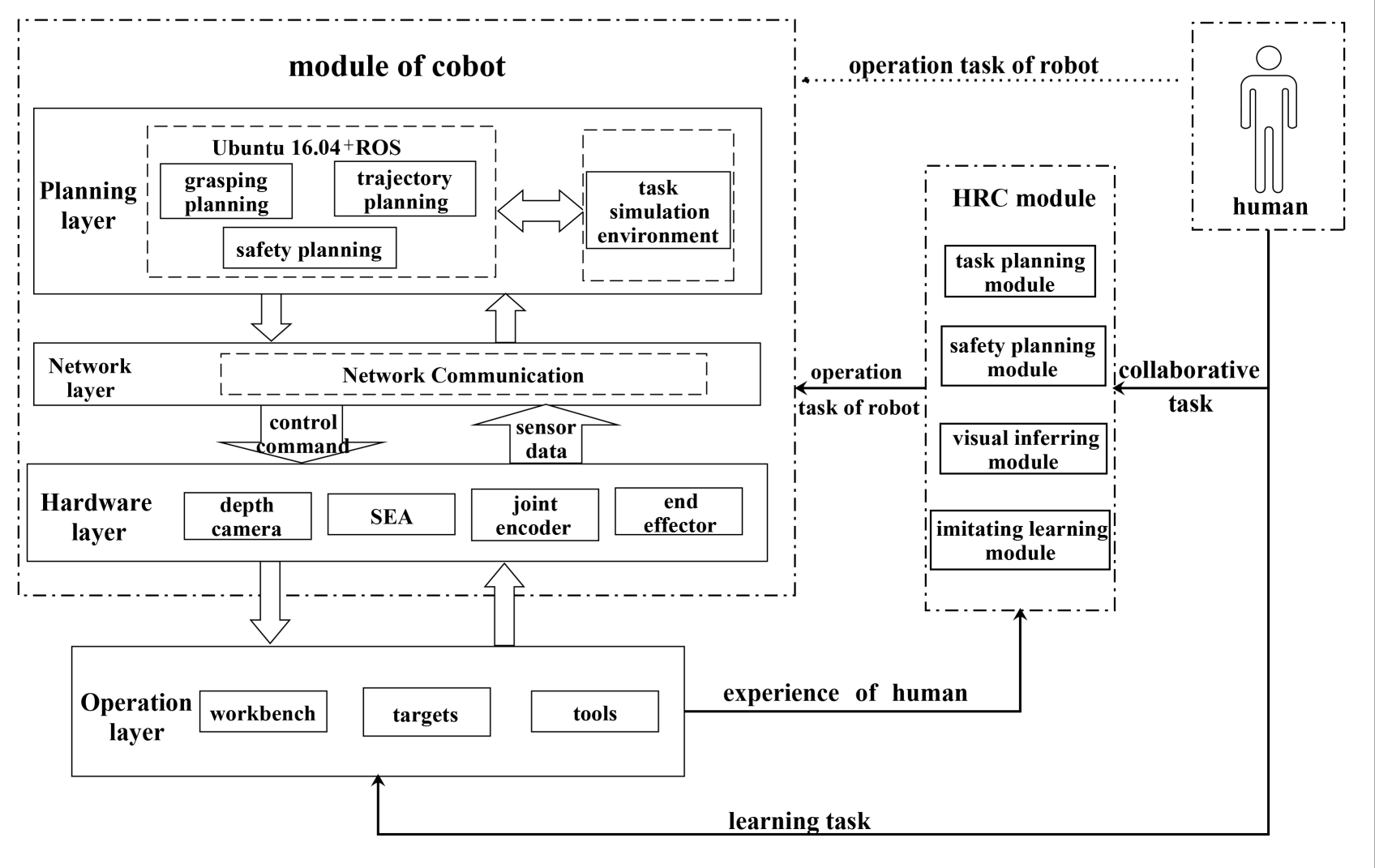

Fig. 2. The prototype system framework of intelligent manufacturing cell based on human-robot collaboration 
scenario and the task process. That can enhance the robustness of the robot for dynamic change. Li, et al [36]also developed a force inferring method to estimate the fuzzy grasping force through visual deformation, which can adjust the close degree of robot via visual data for force sensor-less robot. The two methods can make the robot understand the operation state of robot. It is the precondition for the adjustment of robots in the collaborative tasks.

In order to improve the robot' efficiency of adjustment when dealing with new tasks, this paper developed the HRCIMC. The HRC-IMC was designed to learn the operation experience of human via imitating learning module when dealing with new tasks. And in the collaborative tasks, robot can understand the task state so as to adopt human actions according to sense of vision and force. Besides, robot can ensured the safety of human initiatively through the estimation of safety state. All in all, the HRC-IMC can provide a more efficiency, safety and intelligent system for the manufacturing industry. Moreover, it not only meet the demands of short run production and personalized customization of manufacturing industry of consumer goods, but also can be applied to other manufacturing domain. In addition, the rest parts of this paper are organized as follows. Section 2 describes the modules of the HRC-IMC. Then experiments are set in Section 3. And the results of the experiments are discussed in Section 4. In the end, Section 5 outlines the conclusion.

\section{The intelligent manufacturing cell based on human- robot collaboration}

The cobot Baxter is used as the fundamental hardware of the HRC-IMC. As in known, there are 7 degrees of freedom for each arm of the Baxter robot. And each joint of the robot is equipped with the SEA to estimate the joint torque. Besides, the repeated positioning accuracy of the robot is $5 \mathrm{~mm}$. In addition, the HRC-IMC integrates the tasking planning technology and the visual inferring algorithm of our previous work. With these algorithms, the HRC-IMC can understand the task state and estimate the fuzzy grasping force via visual information. Furthermore, the imitating learning method is employed in the HRC-IMC to make the robot learn from the operation experience of human. That enhances the intelligence of the HRC-IMC by combing the intelligence of human and the operational ability of the robot. Also, the safety planning module can ensure the safety of human in the human-robot co-exist space.

The prototype system framework of HRC-IMC is shown as Fig. 2. The HRC-IMC is mainly composed of three part$\mathrm{s}$, namely, cobot module, human and HRC module. And the functions of HRC-IMC are realized by the planning layer, the network layer, the hardware layer, the operation layer and the HRC module. Furthermore, the layers and modules of HRCIMC are described in detail in the following paragraphs.

Planning layer-according to the operation task commands of cobots, subtasks can be carried out via the planning layer, such as grasping planning, motion planning, dynamic planning, self-collision avoidance planning, collision avoidance planning in Cartesian space and safety planning. This layer is an important module which can decode the control commands into the joint moving of the robot. Besides, all of the commands are published via the controller of ROS. So does the feedback of the sensor data. The motion planning results of the planning layer are also sent to the task simulation environment to verify the planning results before the operation.

Network layer-this layer is designed to realize the transmission between the control system and the hardware system of the robot, such as video feedback, control commands, image processing information, auxiliary information of robot and so on.

Robot hardware layer mainly consists of vision sensor, series elastic actuator (SEA), joint encoder and end-effector. The function of this layer can execute operation commands. And the original data of operation execution can be obtained via sensor information data. Then this data in terms of operation execution are sent to the planning layer.

Operation layer includes workbench, all kinds of objects and tools. It can be regarded as a part of the automatic production line or a station.

HRC module-it is composed of the task planning module, safety planning module, visual inferring module and imitating learning module. And the main function of the HRC module is to conduct the planning of human-robot cooperation tasks and the imitating learning of new tasks.

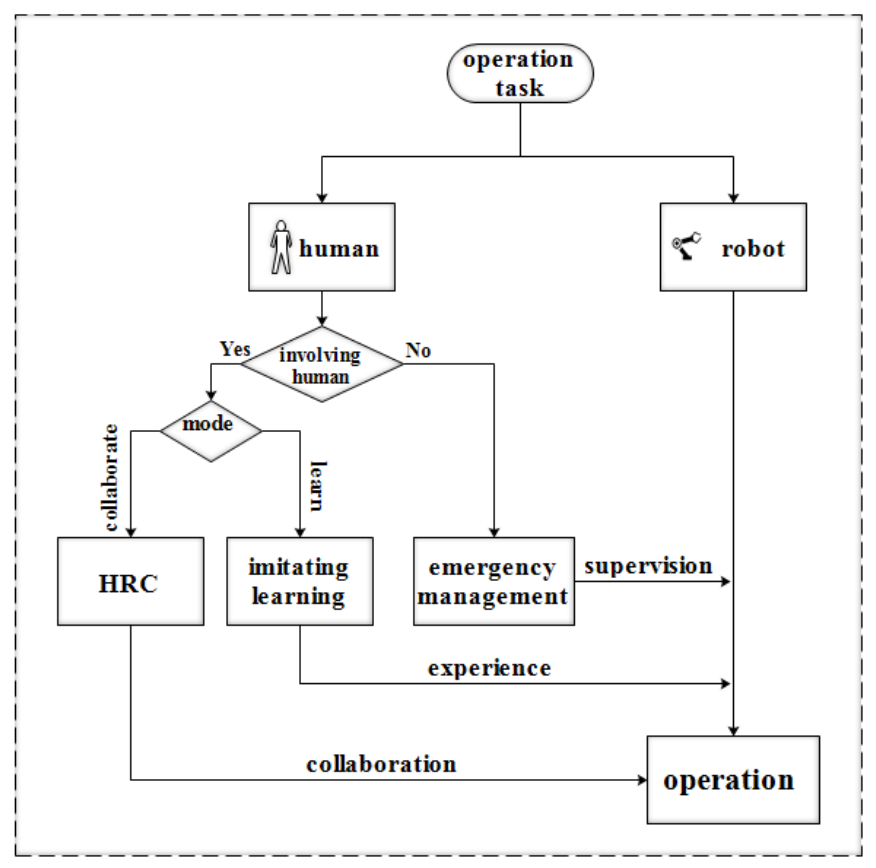

Fig. 3. The role of human in the prototype system

Human-the main functions of man in the HRC-IMC are shown in Fig. 3. Firstly, if it is not necessary for human to directly participate in the task, human will act as the task command publisher and supervisor to monitor the execution of the task. And human should also make emergency management if emergencies occur. Secondly, the tasks involving humans can be divided into two groups, namely the HRC tasks and the imi- 
tating learning tasks. In the HRC tasks, human is demanded to conduct cooperative tasks along with robots. Besides, for some new tasks, humans can share their operating experiences with robots. And with the imitating learning module, human experiences can be learned by robots through deep learning network. Then a supervised learning model of human experience can be obtained and applied for planning of robots when dealing with same tasks or objects. In this way, robots can realize the rapid adjustment of the task. Moreover, with digitizing the human intelligence, the intelligence of HRC-IMC can be improved. So does the manipulation performance of the robot.

\subsection{The development of the modules of the HRC-IMC proto- type system}

\subsubsection{Task simulation environment}

The module of task simulation environment is shown as Fig. 4. In order to establish a visual virtual operating environment, Gazebo is employed to model the three-dimensional task space of robots and operational scenario. And the controller of robots is built based on ROS to ensure the simulation environment interact with the robot in the real physical environment. Besides, the modeling process above mainly involves simulation environment modeling and kinematics modeling. The 3D model of the robot and the object is used to build the corresponding URDF model. And the simulation environment of the shoe-making scene as shown in Fig. 4 is built according to the file format of Gazebo physical simulation engine.

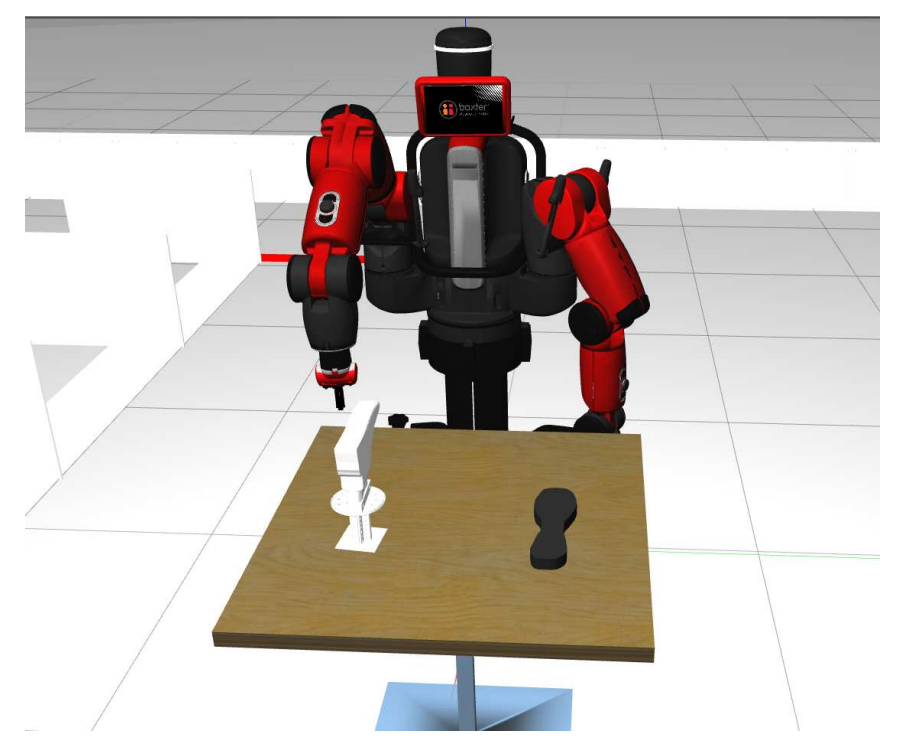

Fig. 4. The Gazebo simulation environment for the prototype system

Based on the robotic kinematics relationship defined in URDF model, kinematics and dynamics library (KDL) was used to generate kinematics chain. The kinematics model was carried out by referring to equations (1)-(5).

For one joint, if the joint' coordinates matrix at time $t_{0}$ is $P_{t 0}$, there are joints angle matrix $\theta_{t 0}$ and seeds angle matrix $\theta_{0}$ which make the formula (1) and (2) true.

$$
P_{t 0}=f\left(\theta_{t 0}\right)
$$

$$
P_{t 0}=f\left(\theta_{0}\right)+J\left(\theta_{0}\right)\left(\theta_{t 0}-\theta_{0}\right)+\text { higher }- \text { order }- \text { terms }
$$

Formula (2) is the Taylor series expansion of formula (1). And the higher-order-terms in formula (2) is the higher order terms of Taylor series expansion. In addition, $J$ stands for jacobian matrix which can be express in detail via formula (3).

$$
J=\left[\begin{array}{llr}
\frac{\partial f_{k 1}}{\partial \theta_{1}} & \cdots & \frac{\partial f_{k 1}}{\partial \theta_{n}} \\
\vdots & \ddots & \vdots \\
\frac{\partial f_{k m}}{\partial \theta_{1}} & \cdots & \frac{\partial f_{k m}}{\partial \theta_{n}}
\end{array}\right]
$$

If higher-order-terms in formula (2) is ignored, $\theta_{t 0}$ can be calculated through formula (4).

$$
\theta_{t 0}=J^{-}\left(\theta_{0}\right) P_{t 0}+\theta_{0}
$$

Formula (2)-(4) is a classical numerical recursion algorith$\mathrm{m}$. And the interpolation termination conditions is shown as formula (5).

$$
\frac{\left|f\left(\theta_{t k}\right)-f\left(\theta_{t(k+1)}\right)\right|}{\left|f\left(\theta_{t k}\right)\right|} \leq \varepsilon
$$

$\varepsilon$ represents threshold value for interpolation search.

\subsubsection{Planning module}

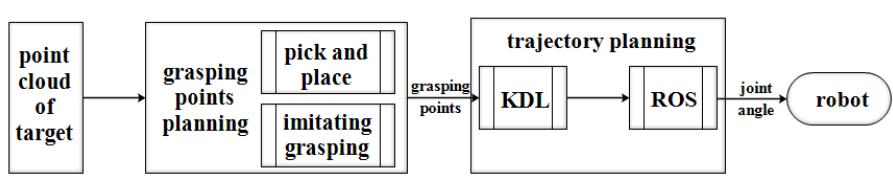

Fig. 5. The process of grasping planning

The planning module mainly realize kinematics planning, including grasping planning, motion planning and safety planning. The grasping planning involves grasping point planning and grasping path planning. The process of grasping planning is shown in Fig. 5.

As shown in Fig. 5, there are two modes of grasping point selection, namely, imitating grasping mode and envelopmen$\mathrm{t}$ model mode. The envelopment model in this paper is realized by the pick-and-place module which is mainly used for the grasping point planning of the simple geometric shapes, such as cylinder, cuboid and so on. The imitating grasping mode is the main grasping point planning method of the HRC-IMC. In this mode, the trained priori model of human grasping for the same object is applied to generate grasping points for the subsequently grasping path planning of robot. And in this process, the pose and semantic information of the objects are set as the input of the priori model. Besides, the path planning for grasping is conducted by trajectory planning module in the planning layer. And trajectory planning is mainly realized by combining the KDL library and ROS. With the functions in the KDL library, path points are fitted into paths with trapezoidal wave velocity characteristics, which are then converted into ROS path point format. Finally, the trajectory tracking control is realized based on the motion controller of ROS. 


\subsubsection{HRC module}

The network module realizes the information communication between the control system and the robot, which is the basis of the task command sending to the robot and the feedback of the data of sensors. In this paper, the data need to be exchanged including real-time point cloud and image data, control command data and auxiliary information data, such as joint angle and SEA force information of robot. Socket communication protocol is used in this paper to establish the connection between the control system and the robot, which will not be described in detail here.

The HRC module is mainly composed of the task planning module, safety planning module, the visual inferring module and the imitating learning module. The visual inferring module mainly estimates the fuzzy hardness according to the visual information. And this module was mainly developed according our previous work[36]. Besides, the function of the task planning module is mainly realized by the method proposed in ref.[35]. The imitating learning module is developed based on ref.[37]. The safety planning module in this paper is mainly developed for the contact maintenance scenario of cobots. At present, there is only human-machine safety planning function based on vision and force fusion. Since the robot control system is developed under the framework of ROS, in order to facilitate the information transfer between different modules, the HRC module is developed in the form of ROS package. And the data information between modules is transmitted in the form of topic.

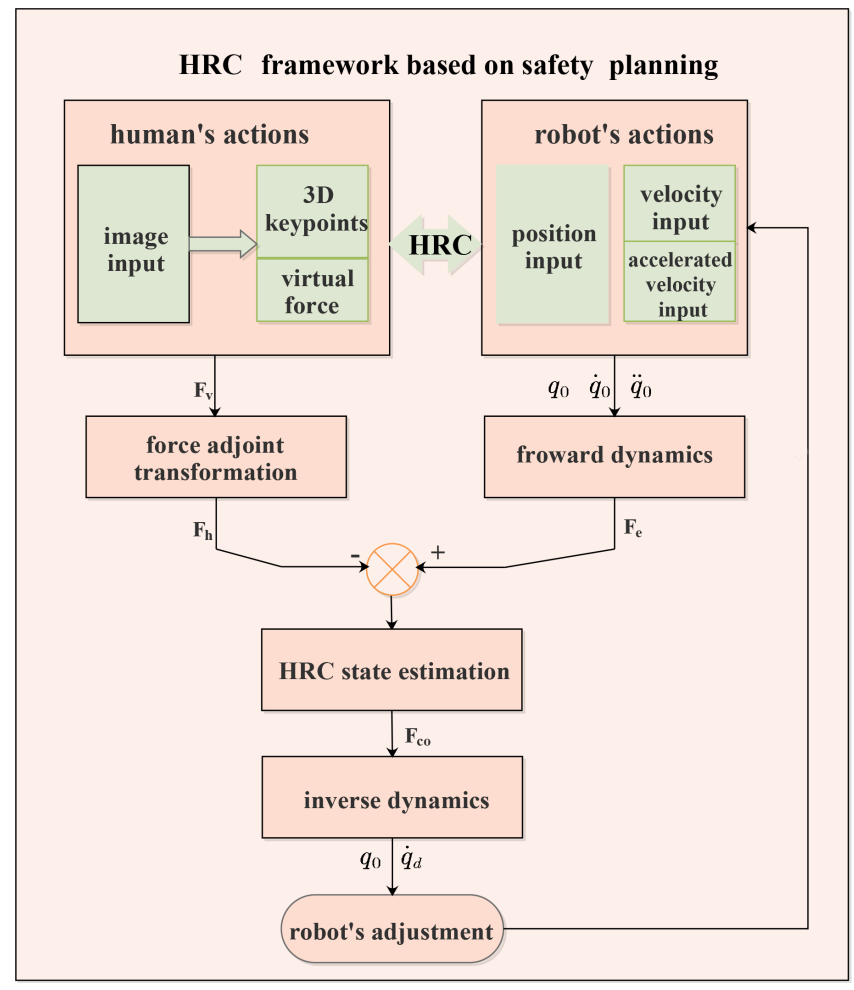

Fig. 6. The HRC framework based on safety planning

As shown in Fig. 6, the human-inspired collaboration strategy was employed in the HRC module. And the HRC s- trategy was realized through safety planning based on humanrobot distance. With the HRC strategy, human's actions can be extracted in RGB image. After the corresponding depth information of keypoints being extracted, the 3D keypoints of human are estimated. Then the minimum distance between human and robot can be obtained with the help of their bounding boxes. And this minimum distance is converted to virtual force through formula (6) which can be exerted on the robot's end effect after adjoint transformation.

$$
F_{v t}=\frac{F_{e}}{1+\left(\frac{d_{t}-k_{b}}{k_{a}}\right)^{2 k_{c}}}
$$

$F_{e}$ in formula (7) represents the force of the robot at the endeffector; $d_{t}$ is the minimum distance between human and robot at time $\mathrm{t} ; F_{v t}$ stands for the virtual force at time $\mathrm{t} ; k_{a}, k_{b}$ and $k_{c}$ stand for the adjustment parameters of virtual force.

Furthermore, the superposed effect on end effort, named $F_{c o}$, is calculated directly according to the forward dynamics and other input elements. The $F_{c o}$ and its duration $t$ are two paramount parameters for the estimation of safety state. Based on the estimated safety state valued as $s$, the robot can conduct safety planning via inverse dynamic according to formula (7)(13), taking the interrupt position points $q_{c}$ and interrupt time points $t_{i}$ into consideration.

$$
\begin{gathered}
\tau=M(q) \ddot{q}+C(q, \dot{q})+G(q) \\
F=V(q) \ddot{x}+u(q, \dot{q})+p(q)
\end{gathered}
$$

$M(q)$ in formula (7) is generalized mass matrix; $V(q)$ in formula (8) stands for inertial matrix; $C(q, \dot{q})$ represents centrifugal and Coriolis force vector in formula (7); $u(q, \dot{q})$ has the same meaning with $C(q, \dot{q})$. Furthermore, $G(q)$ and $p(q)$ are gravity vectors in formula (7) and (8) respectively. $x$ is the desired position at end-effector. And $q$ is the desired joint angles.

Virtual displacements of each joint are set as $\partial q$. And the displacement of robot's end effector is set as $D$. Accelerated velocity of robot's end effector and joints, which is subject to force on robot's end effector, can be calculated through formulas (9)-(13) according to the principle of virtual work.

$$
\begin{gathered}
W=\tau^{T} \partial q=F^{T} D \\
\tau=J^{T} F \\
\left\{\begin{array}{l}
V(q)=J^{-\dagger T}(q) M(q) J^{-\dagger}(q) \\
u(q, \dot{q})=J^{-\dagger T}(q) C(q, \dot{q}) J^{-\dagger}(q)-V(q) a_{r}(q, \dot{q}) \\
p(q)=J^{-\dagger}(q) G(q)
\end{array}\right. \\
\left\{\begin{array}{l}
\ddot{q}=M^{-1}(q) J^{T} F \\
\ddot{x}=J(q) M^{-1}(q) J^{T} F
\end{array}\right.
\end{gathered}
$$

$J$ is jacobian matrix of robot; $J^{-\dagger}$ stands for pseudo-inverse of $J$.

In addition, the position control quantity $x_{0}$ in impedance controller can be calculated through formula (21) when the external force on robot's end effector is $F_{t}$ and the desired position is $x_{d}$ at time $\mathrm{t}$.

$$
M\left(\ddot{x}_{d}-\ddot{x}_{0}\right)+C\left(\dot{x}_{d}-\dot{x}_{0}\right)+G\left(x_{d}-x_{0}\right)=-F_{t}
$$




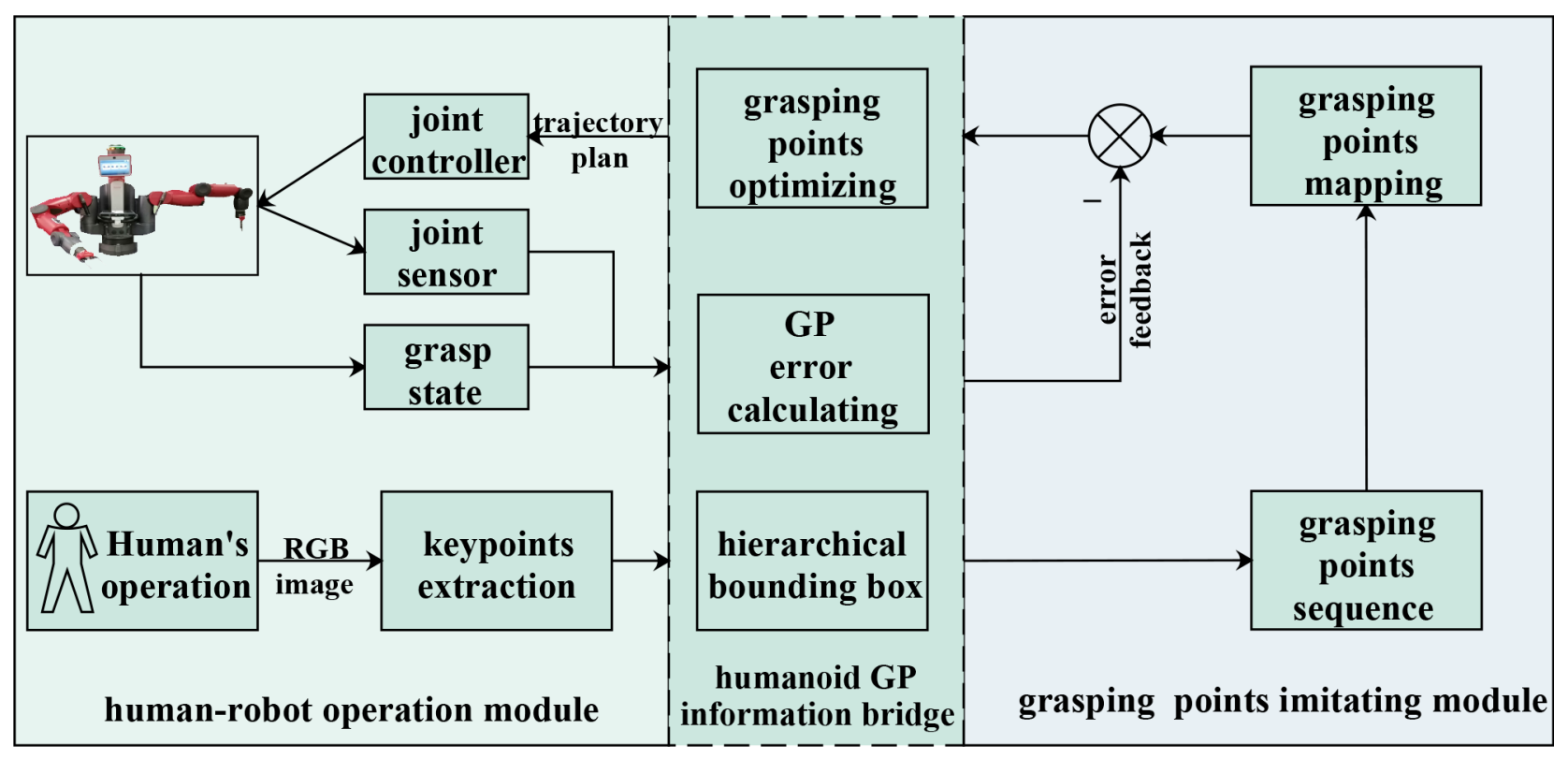

Fig. 7. The imitating learning framework in HRC module

Then the desired new joints position $q_{d}$ and joints velocity $\dot{q}_{d}$ can be calculated based on $x_{d}$ and $\dot{x}_{d}$. Subsequently $q_{d}$ and $\dot{q}_{d}$ were sent to robot control module. In this way, robot can decreases its velocity until stopping along with the approaching of human. More importantly, the task would be continuous if the state switches into the collaborative-move. This slow down and continuous mode can guarantee both the safety of human and the efficiency of execution.

The grasping planning of robots involving lots of mathematical modeling works when dealing with new tasks or new targets. So the study of imitating learning in the HRC module focused on the GP planning of robots. In this way, robot can adjust quickly through learning from human instead of mathematical modeling. Besides, the framework of imitating learning module of the HRC module was shown as Fig. 7. In Fig. 7, the control principle of robots' imitating human's operation can be divided into three parts according to their functions in the imitating process. Human-robot operation module involves the input of human's operation extraction and output of robots' imitating operation; the humanoid GP information bridge, which is composed of hierarchical bounding box for hands' action position tracking, grasping points optimizing and GP error calculating for feedback via calculating GP error. Firstly, in humanrobot operation, keypoints extraction is conducted from RGB image which is in terms of human's operation actions. And these keypoints are converted to array and published to the humanoid GP information bridge via ROS master. Then they can be subscribed by both robots and users in control system. In addition, hierarchical bounding boxed of hands and objects being built according to the hands' keypoints, GP sequence are estimated through FCL by intersection of closest points calculation and send to grasping points imitating module. Moreover, the GP mapping is introduced to overcome the problem resulting from different structures between robots' gripper and humans' hands in this module. Finally, the optimized GP are applied to trajectory planning for robotic grasping operation through humanoid GP information bridge.

\subsection{The fusion mechanisms of visual and force in the HRC- $I M C$}

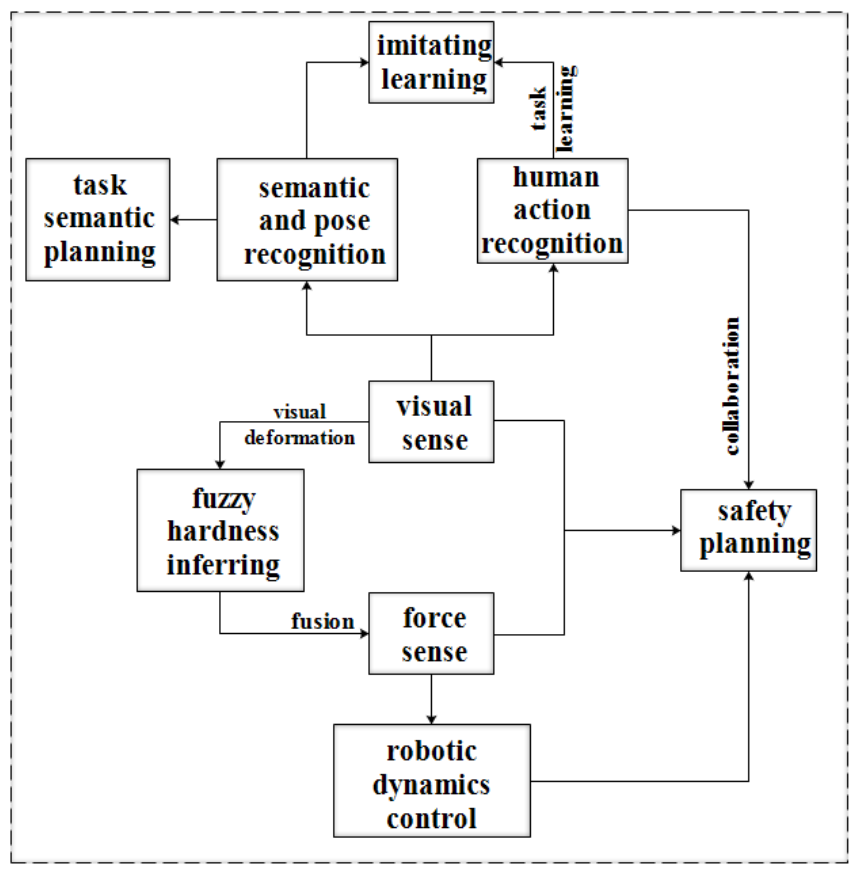

Fig. 8. The fusion mechanisms of visual and force in the HRC-IMC

The fusion of vision and force sense mechanism in the prototype system of HRC-IMC is shown in Fig. 8. As shown in Fig. 8, the vision in this paper is mainly used in three domain: a) estimation of object deformation information; b) recognition of object' semantics and pose; c) recognition of human's actions. Since the force sensor used in this paper is only SEA, the 
actual force perception information is only joint force information. the other forces data, such as grasping force and end-effort force, can only be obtained by calculation. The mechanism of force induced by vision is mainly embodied in the fuzzy hardness inference in the visual inferring module. According to the content of ref. [36], the supervised learning mechanism built by LSTM network can realize grasping force adjustment by transforming visual deformation into fuzzy hardness.

In addition, after the object's semantic and pose information being recognized via visual information, the target semantic matrix generated by the task planning can be used to realize the autonomous modeling of the target and obstacle. That can help robot realize the understanding of task and the autonomous adjustment in the process of the task. Besides, when human actions are recognized, robots can conduct imitating learning according to the semantic and pose information of the objects. Then the robot can quickly obtain the operated ability of new tasks. Moreover, the human actions can also be applied for the estimation of the safety state according to the visual and force information in the cooperative operation. In this way, the safety of human can be ensured in the cooperative task. The aforementioned are the fusion mechanism of vision and force in this paper. As the intelligent requirements of cobots become higher and higher, it is difficult to realize intelligent control only by relying on single information channel. Therefore, the fusion mechanism of vision and force based on human operation mode can meet the demands of different task. That may be one of the effective ways to realize intelligent control of HRC.

\subsection{The hardware and software environment for HRC-IMC}

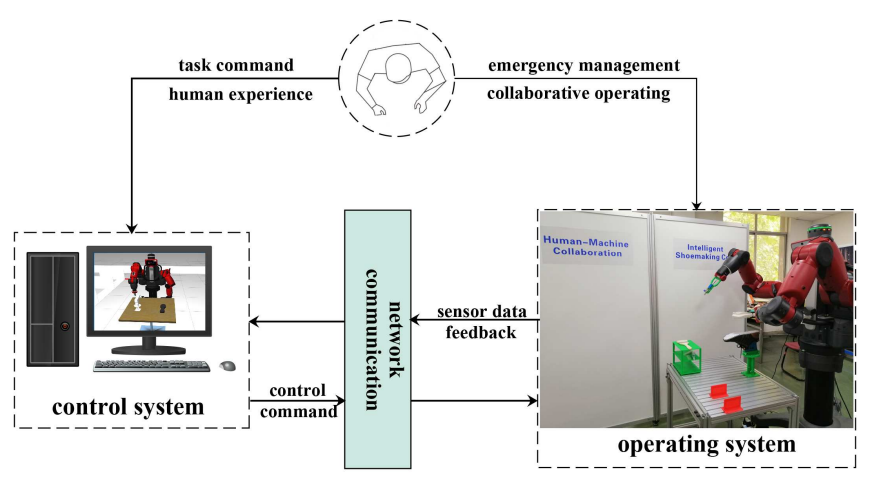

Fig. 9. The system structure of HRC-IMC

The structure of HRC-IMC developed in this paper is shown in Fig. 9. The hardware and software environment of the HRC-IMC is shown in table 1. The hardware of the system mainly includes: Baxter cooperative robot from Rethink Company, two-finger electric gripper, Kinect V1 depth camera, a laptop computer, common tools and operating objects in shoe production line, test platform and tooling and fixture. The computer is configured with an Intel $17-7700$, a $2.8 \mathrm{GHz}$ CPU and $8 \mathrm{~GB}$ of RAM.

The system is built in Ubuntu16.04 system environment with ROS Kinetic architecture. The Qt is employed as the main development platform. Besides, the 3D models of robot, target and environment are modeled and generated by SolidWorks 2016, which are converted by URDF plug-in. The network communication function is realized by Socket programming. Baxter SDK is used to conduct robot control and joint information feedback. The OpenCV and PCL libraries are used for image and point cloud processing. Besides, the installation address of the above visual processing library file is added to the cMakeList file of the Qt project package.

\section{Experiment}

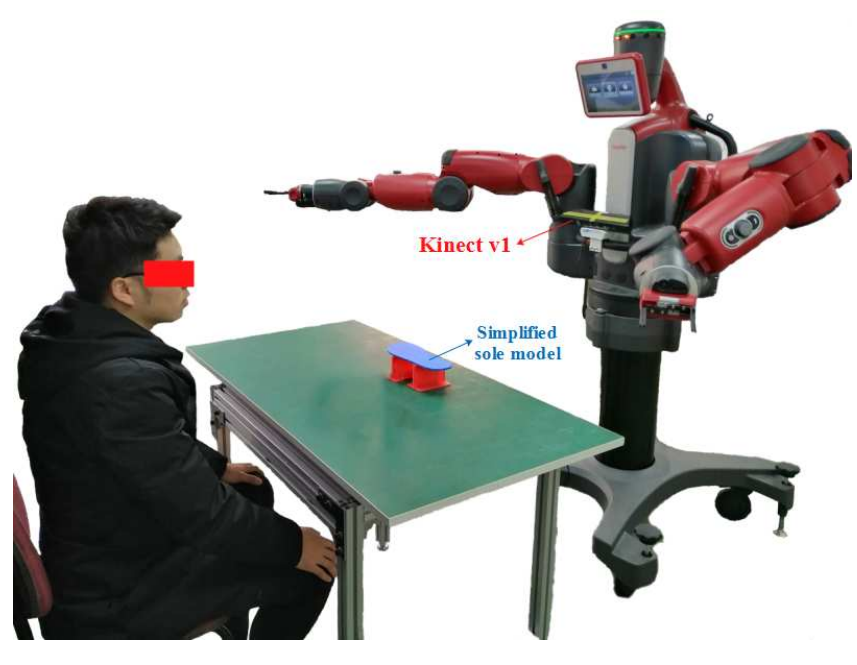

Fig. 10. The experimental scene

A imitating grasping experiment was conducted to evaluate the efficiency of task adjustment of the HRC-IMC above. As shown in Fig. 10, the main equipments applied to conduct the experiment are a Baxter robot with parallel gripper, a Kinect v1 camera. Moreover, a laptop with a graphics card of GTX1060

Table 1: The hardware and software environment for HRC-IMC

\begin{tabular}{|c|c|c|}
\hline \multirow{3}{*}{ Hardware } & Computer & Intel i7-7700 2.8GHz, 8G internal storage, GTX 1060 \\
\cline { 2 - 3 } & Display system & CRT monitor(120Hz refresh rate) \\
\hline \multirow{5}{*}{ Software } & Programming languages /tools & C++, Python/QT \\
\cline { 2 - 3 } & System architecture & Ubuntu 16.04 + ROS Kinetic \\
\cline { 2 - 3 } & Packages for image processing & OpenCV 3.4.2+PCL 1.8.0 \\
\cline { 2 - 3 } & Machine Learning Framework & Tensorflow 1.4.1 \\
\cline { 2 - 3 } & Robotic drive package & Baxter SDK1.2 \\
\cline { 2 - 3 } & Other third-party libraries & KDL+FCL+Openpose \\
\hline
\end{tabular}


and Baxter sdk1.2 environment was used for image processing and robot's motion planning. In addition, a simplified sole model was used to conduct grasping operations. Since the grasping points selection of sole changes when task switches, the efficiency from modeling to grasping points selection were tested via imitating grasping and Pick-and-Place module of Moveit. In order to conduct contact points estimation between hands and objects according to geometric intersection, PCL, FCL and TensorFlow were also applied in this experiment.

Furthermore, two groups experiments were conducted to verify the feasibility of the task adjustment via the HRC-IMC. One-hand grasping experiments were used to test the feasibility grasping points imitating from human. And two-hands operation was applied to imitate dual-arm operation scene.

\section{Result and discussion}

\subsection{One-hand grasping}

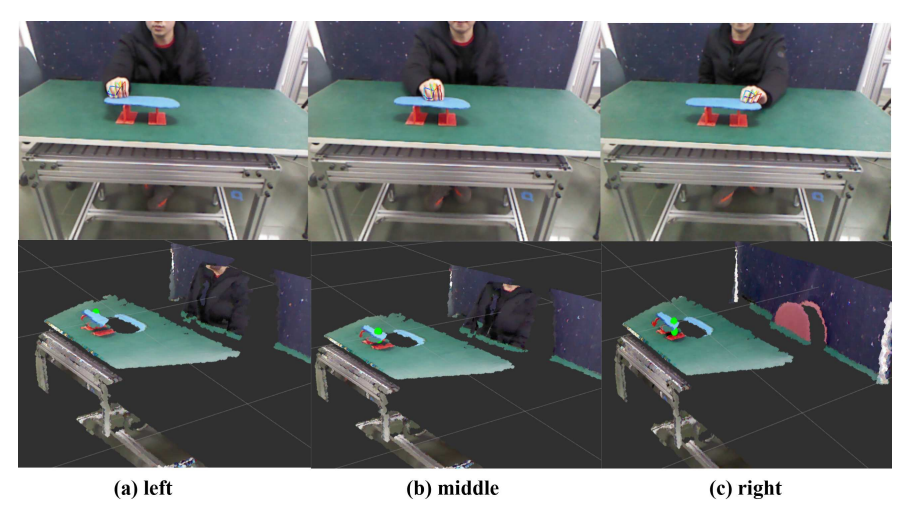

Fig. 11. One-hand limiting grasping result for shoe sole

One of the GP point clouds for shoe sole is shown in Fig. 11. And Fig. 12 is three grasping trajectories for three different GP when the shoe sole model remains in a same position. As shown in Fig. 12 (b), the grasping RPY degree at start position and goal position are set as same value so as to minimize path planning failure rate of ROS resulting from different grasping RPY setting. The invariant obstacle position and small change for three GP contribute to the nearly same change of RPY along trajectory in Fig. 12 (b). As shown in figures, the GP imitating human are generated as point cloud (green) and combined with objects' points clouds (blue). Moreover, the success rates of 60 times repeated experiments and the efficiency of modeling are listed in table 2. Furthermore, for one hand's grasping operation on flat and long object like shoe sole, the probability of GP lying in the left, middle or right is almost same and would not introduce negative factor to human for success grasping. As shown in table 2, the success rate for grasping sole is $70 \%$, which is $10 \%$ higher than that of Pick-and-Place module. The two reason which makes the success rate decrease for sole model can be explained through Fig. 12 (a). For baxter robot, the kinematic chain in OMPL of ROS for trajectory planning is from link torso to link gripper. In addition, the origin of link gripper coordinates is at the center of each gripper. And the

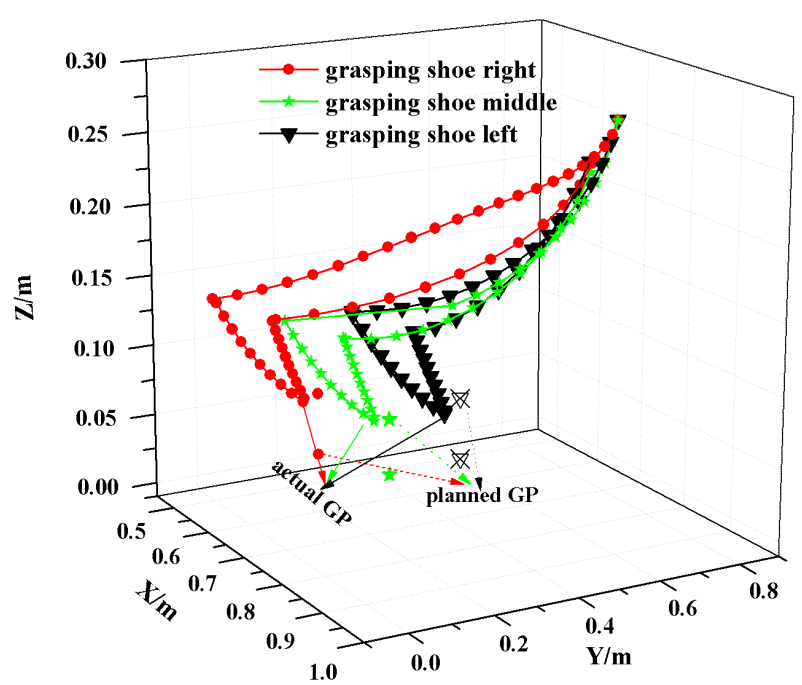

(a)position of gripper
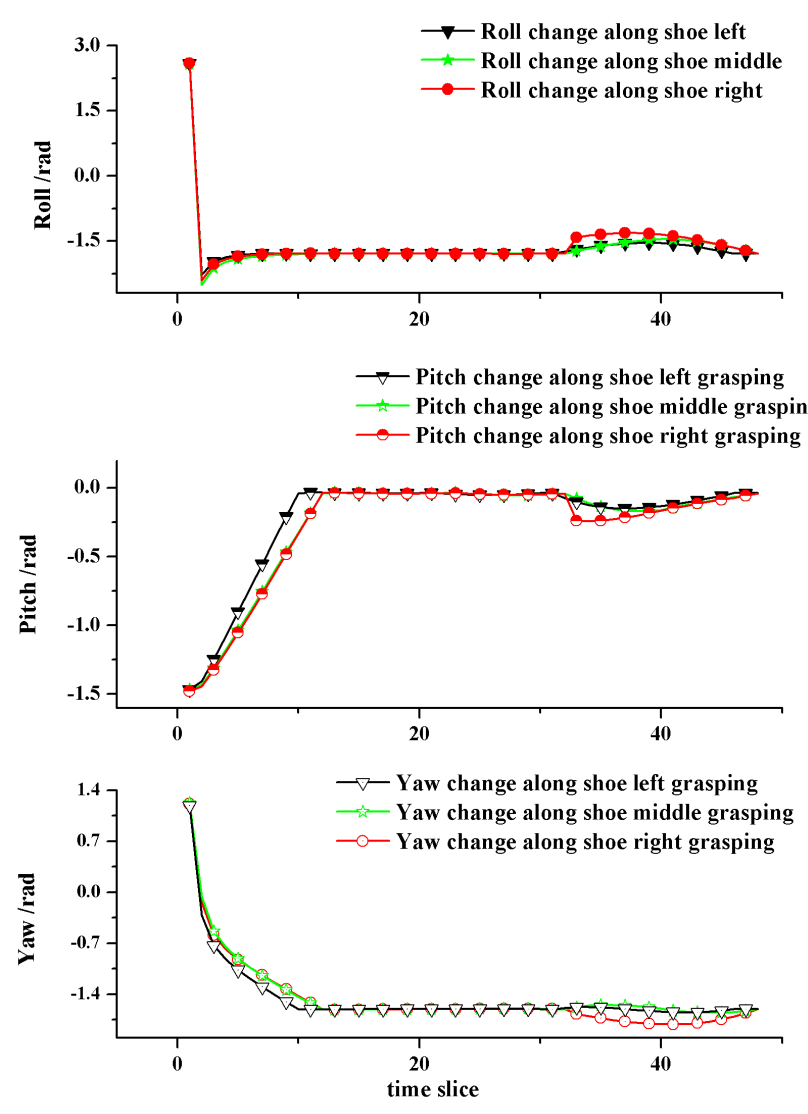

(b)RPY

Fig. 12. GP and trajectory planning for lateral hip

length of each gripper is $0.011 \mathrm{~m}$. The maximum error in $\mathrm{Y}$ axis is thereby $0.055 \mathrm{~m}$ which almost has no effect on grasping success rate. If the error in $\mathrm{Y}$ axis approaches or surpasses $0.0055 \mathrm{~m}$, the slippage may occur when conduct grasping operation on shoe sole. And this phenomenon is the main reason for grasping failure because of the relatively low accuracy for baxter robot. The other reason leading to grasping failure is 
the trajectory planning problem for ROS in grasping process, especially when taking obstacle avoidance into consideration. Furthermore, as shown in Fig. 12(a), the key factor to grasping success rate of shoe sole model is that the grasping force for holding the objects along the trajectory back to start pose. And the grippers with sucker can enhance the success grasping rate.

Table 2: success rate of One-hand grasping for different objects

\begin{tabular}{|c|c|c|}
\hline Methods Data & success rate & efficiency $(/ \min )$ \\
\hline Pick-and-Place & $60 \%$ & 35 \\
\hline HRC-IMC & $70 \%$ & 20 \\
\hline
\end{tabular}

As aforementioned, the efficiency in table 2 is the time consuming from geometric modeling to the obtaining the grasping points results. As shown in table 2, the efficiency from setting the simplified model to the accomplishment of grasping points is 35 minutes. It is 20 minutes for the imitating learning module. And the efficiency of adjustment can be increased $42.9 \%$ when using the imitating learning module of the HRCIMC. That demonstrated the superiority of the HRC-IMC.

\subsection{Two-hands grasping}

As shown in Fig. 13 (a), dual-arm GP imitating is the development from one-hand grasping in Fig. 12. The grasping force adjustment in scene of Fig. 12 can be avoid via two hands operation. Moreover, the Fig. 13 (b) and (c) can indicate that the grasping process can be divided into independent operations of left arm and right arm before move to grasping position. But in Fig. 13 (b), the trajectories of dual-arm after actual GP is synchronous. And the bottleneck for increasing dual-arm grasping success rate lies here. Although the experiments can verify the feasibility of GP imitating human's two-hands operation, the path planning for dual-arm moving with end effort remaining grasping pose to target pose is still key limiting factor for improving grasping success rate.

\section{Conclusion}

This work aims to enable robots in the production line to realize quickly adjustment in flexible manufacturing, which meet the demands of the short run production. And the HRCIMC was presented which can combined the operational experience of human and the operational capacity of robot, rather than adjusting different mathematical model for different tasks. With the HRC-IMC, critical problems of robots involving the evaluation of task state and the safety planning in task process were addressed. Moreover, the understanding of the scene and task for robots can promote the state evaluation in the tasklevel. That can improve the efficiency of HRC. Besides, the experimental results indicated that the the efficiency of task adjustment of HRC-IMC can be increased $42.8 \%$ than that of Moveit. Therefore, in the short run production, the HRC-IMC can be used as a more intelligent and efficient substitute for the traditional automatic manufacturing cell.

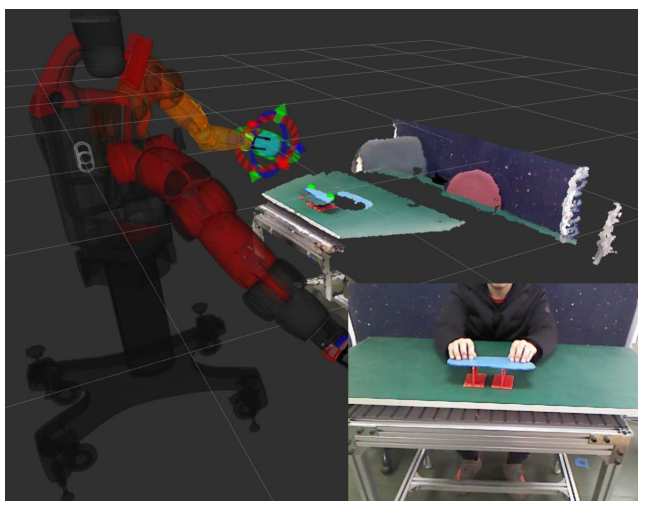

(a)GP for two hands

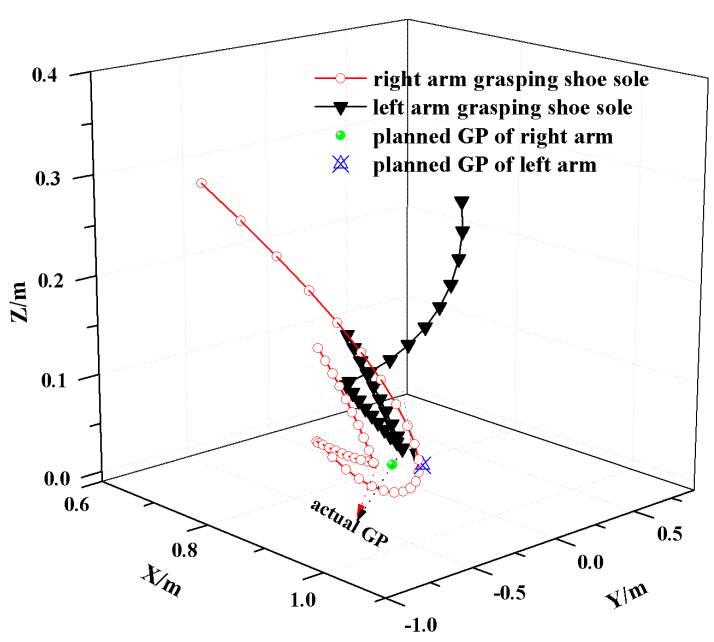

(b)trajectory of robot's dual arm grasping
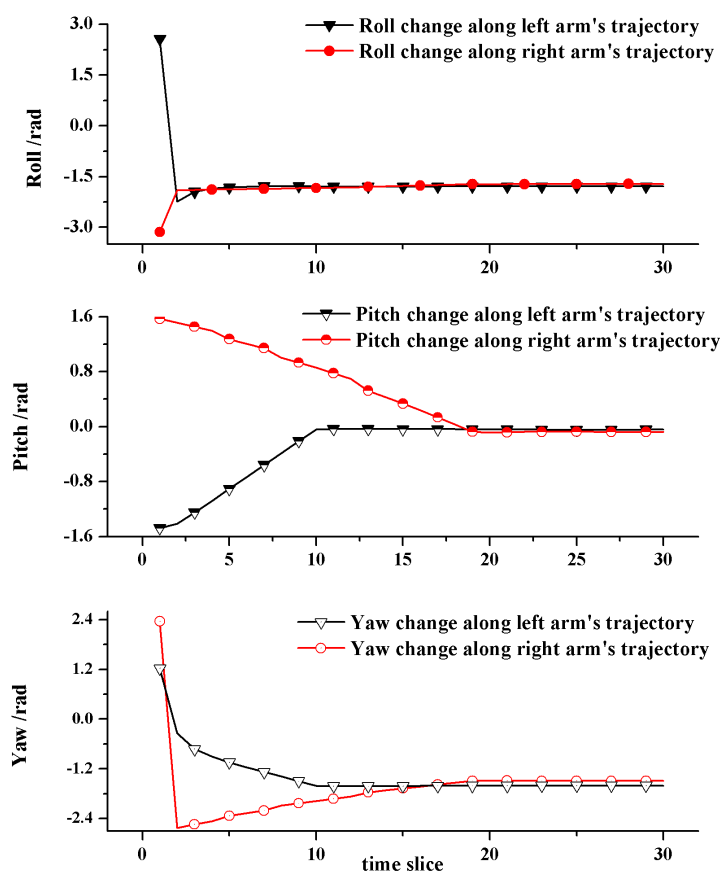

(c)RPY change along robot's trajectory

Fig. 13. Two-hands limiting grasping for sole 


\section{Declarations}

\subsection{Acknowledgments}

We acknowledge the support received from the HUST \& UBTECH Intelligent Service Robots Joint Lab.

\subsection{Conflict of interest statement}

We declare that we have no financial and personal relationships with other people or organizations that can inappropriately influence our work. There is no professional or other personal interest of any nature or kind in any product, service and/or company that could be construed as influencing the position presented in, or the review of, the manuscript entitled.

\subsection{Availability of data and material}

All data generated or analysed during this study are included in this published article.

\subsection{Code availability}

Not applicable.

\subsection{Ethics approval}

Not applicable

\subsection{Consent to participate}

Informed consent was taken from the family to participate in the study.

\subsection{Consent for publication}

Informed consent was taken from family to publish the data in an anonymized manner.

\subsection{Authors' Contributions}

Shuai Zhang and Shiqi Li conceived and designed the study. Shuai Zhang and Xiao Li performed the experiments. Shuai Zhang wrote the paper. Shuai Zhang, Shiqi Li and Haipeng Wang reviewed and edited the manuscript. All authors read and approved the manuscript.

\section{References}

[1] R. Y. Zhong, X. Xun, E. Klotz, and S. T. Newman. Intelligent manufacturing in the context of industry 4.0: A review. Engineering, (3):616-630, 2017.

[2] J. Zhou, P. Li, Y. Zhou, B. Wang, and L. Meng. Toward new-generation intelligent manufacturing. Engineering, 4(4):11-20, 2018.

[3] Henry Zhu, Abhishek Gupta, Aravind Rajeswaran, Sergey Levine, and Vikash Kumar. Dexterous manipulation with deep reinforcement learning: Efficient, general, and low-cost. In 2019 International Conference on Robotics and Automation (ICRA), pages 3651-3657. IEEE, 2019.

[4] A. Karami, H. Sadeghian, M. Keshmiri, and G. Oriolo. Hierarchical tracking task control in redundant manipulators with compliance control in the null-space. Mechatronics, 55:171-179, 2018.

[5] L. Sheng, L. Bao, and W. U. Peng-Fei. Application of heuristic approaches in the robot path planning and optimization: A review. Electronics Optics $\mathcal{E}$ Control, 2018.

[6] M. Raessa, Jcy Chen, W. Wan, and K. Harada. Human-in-the-loop robotic manipulation planning for collaborative assembly. 17(4):1800-1814, 2020 .
[7] Xi, Vincent, Wang, Zsolt, Kemny, Jzsef, Vøncza, Lihui, and Wang. Humancrobot collaborative assembly in cyber-physical production: Classification framework and implementation. Cirp Annals Manufacturing Technology, 66(1):5-8, 2017.

[8] R. Weitschat and H. Aschemann. Safe and efficient humancrobot collaboration part ii: Optimal generalized human-in-the-loop real-time motion generation. IEEE Robotics and Automation Letters, 3(4):3781-3789, 2018.

[9] S. Music, G. Salvietti, Pbg Dohmann, F. Chinello, and S. Hirche. Humanrobot team interaction through wearable haptics for cooperative manipulation. IEEE Transactions on Haptics, 12(3):350-362, 2019.

[10] Smm Rahman. Cognitive cyber-physical system (c-cps) for human-robot collaborative manufacturing. In 2019 14th Annual Conference System of Systems Engineering (SoSE), 2019.

[11] D. Nunes, J. S. Silva, and F. Boavida. A practical introduction to humanin-the-loop cyber-physical systems. 2018.

[12] G. Schirner, D. Erdogmus, K. Chowdhury, and T. Padir. The future of human-in-the-loop cyber-physical systems. Computer, 46(1):36-45, 2013.

[13] S. K. Sowe, E. Simmon, K. Zettsu, F. D. Vaulx, and I. Bojanova. Cyberphysical-human systems: Putting people in the loop. IT Professional, 18(1):10-13, 2016.

[14] M. Krugh and L. Mears. A complementary cyber-human systems framework for industry 4.0 cyber-physical systems. Manufacturing Letters, 15(PartB):89-92, 2018.

[15] J. Zhou, Y. Zhou, B. Wang, and J. Zang. Humanccybercphysical systems (hcpss) in the context of new-generation intelligent manufacturing. Engineering, 2019.

[16] Iaki Maurtua, A. Ibarguren, J. Kildal, L. Susperregi, and B. Sierra. Human-robot collaboration in industrial applications: Safety, interaction and trust. International Journal of Advanced Robotic Systems, 14(4):110,2017

[17] Ioannis Kostavelis, Andreas Kargakos, Dimitrios Giakoumis, and Dimitrios Tzovaras. Robots workspace enhancement with dynamic human presence for socially-aware navigation. In international conference on computer vision systems, pages 279-288, 2017.

[18] Christoph Sprunk, Boris Lau, Patrick Pfaff, and Wolfram Burgard. An accurate and efficient navigation system for omnidirectional robots in industrial environments. Autonomous Robots, 41(2):473-493, 2017.

[19] Fabrizio Flacco, Torsten Kroeger, Alessandro De Luca, and Oussama Khatib. A depth space approach for evaluating distance to objects. Journal of Intelligent and Robotic Systems, 80(1):7-22, 2015.

[20] Yufan Zhou, Haiwei Dong, and Abdulmotaleb El Saddik. Learning to estimate 3d human pose from point cloud. IEEE Sensors Journal, 99:1$1,2020$.

[21] Haobin Shi, Jialin Chen, Wei Pan, Kaoshing Hwang, and Yiyun Cho. Collision avoidance for redundant robots in position-based visual servoing. IEEE Systems Journal, 13(3):3479-3489, 2019.

[22] Nikolaos Nikolakis, Vasilis Maratos, and Sotiris Makris. A cyber physical system (cps) approach for safe human-robot collaboration in a shared workplace. Robotics and Computer-integrated Manufacturing, 56:233243, 2019.

[23] Azfar Khalid, Pierre T Kirisci, Zeashan Hameed Khan, Zied Ghrairi, Klausdieter Thoben, and Jurgen Pannek. Security framework for industrial collaborative robotic cyber-physical systems. Computers in Industry, 97:132-145, 2018.

[24] Lei Zhu, Zijing Chi, Fan Zhou, and Chungang Zhuang. Dynamic motion planning algorithm in human-robot collision avoidance. In international conference on intelligent robotics and applications, pages 655-666, 2019.

[25] Emanuele Magrini, Federica Ferraguti, Andrea Jacopo Ronga, Fabio Pin, Alessandro De Luca, and Francesco Leali. Human-robot coexistence and interaction in open industrial cells. Robotics and Computer-Integrated Manufacturing, 61(Feb.):1-19, 2020.

[26] Mark Edmonds, Feng Gao, Xu Xie, Hangxin Liu, Siyuan Qi, Yixin Zhu, Brandon Rothrock, and Song-Chun Zhu. Feeling the force: Integrating force and pose for fluent discovery through imitation learning to open medicine bottles. In 2017 IEEE/RSJ International Conference on Intelligent Robots and Systems (IROS), pages 3530-3537. IEEE, 2017.

[27] Tu-Hoa Pham, Nikolaos Kyriazis, Antonis A Argyros, and Abderrahmane Kheddar. Hand-object contact force estimation from markerless visual tracking. IEEE transactions on pattern analysis and machine intelligence, 
40(12):2883-2896, 2017.

[28] Tu-Hoa Pham, Abderrahmane Kheddar, Ammar Qammaz, and Antonis A Argyros. Towards force sensing from vision: Observing hand-object interactions to infer manipulation forces. In Proceedings of the IEEE conference on computer vision and pattern recognition, pages 2810-2819, 2015.

[29] Omid Taheri, Nima Ghorbani, Michael J. Black, and Dimitrios Tzionas. GRAB: A dataset of whole-body human grasping of objects. In European Conference on Computer Vision (ECCV), 2020.

[30] Samarth Brahmbhatt, Chengcheng Tang, Christopher D. Twigg, Charles C. Kemp, and James Hays. Contactpose: A dataset of grasps with object contact and hand pose. arXiv preprint $2007.09545 v 1,2020$.

[31] G. Chang and D. Kulic. Robot task learning from demonstration using petri nets. In RO-MAN, 2013 IEEE, pages 31-36, 2013.

[32] A. Casalino, F. Cividini, A. M. Zanchettin, L. Piroddi, and P. Rocco. Human-robot collaborative assembly: a use-case application - sciencedirect. IFAC-PapersOnLine, 51(11):194-199, 2018.
[33] N. T. Dantam, Z. K. Kingston, S. Chaudhuri, and L. E. Kavraki. Incremental task and motion planning: A constraint-based approach. pages $1-6,2016$.

[34] Dantam N. T., Kingston Z. K., and Chaudhuri S. An incremental constraint-based framework for task and motion planning. The International Journal of Robotics Research, 37(10):1134-1151, 2018.

[35] S. Li, S. Zhang, Y. Fu, H. Wang, and K. Han. Task-based obstacle avoidance for uncertain targets based on semantic object matrix. Control Engineering Practice, 105:104649, 2020.

[36] S. Li, S. Zhang, Y. Fu, Y. Xiong, and Z. Xie. Grasp2hardness: fuzzy hardness inference of cylindrical objects for grasp force adjustment of force sensor-less robots. Intelligent Service Robotics, 14(2):129-141, 2021.

[37] S. Zhang, S. Li, and X. Li. A visual imitating learning algorithm for selection of grasping points for dual-arm robots in intelligent manufacturing industry. Neural Computing and Applications, page submitted, 2021. 